Review

\title{
Current Progress in Cancer Immunotherapies Using Small Molecules Targeting PD-L1 Stability
}

\author{
Macyn Leung, Alexander Pipchuk, Xiaolong Yang *
}

Department of Pathology and Molecular Medicine, Queen's University, 88 Stuart Street, Kingston, Canada; E-Mails: 17mlyl@queensu.ca; 15ap63@queensu.ca; yangx@queensu.ca

* Correspondence: Xiaolong Yang; E-Mail: yangx@queensu.ca

Academic Editor: Tapan K Bera

Special Issue: Molecular Cancer Therapeutics

OBM Genetics

2021, volume 5, issue 1

doi:10.21926/obm.genet.2101127
Received: January 11, 2021

Accepted: March 17, 2021

Published: March 24, 2021

\begin{abstract}
PD-L1 is an immune checkpoint protein that is frequently overexpressed by the cells in the tumor microenvironment. PD-L1 binds to PD-1 present on the activated antitumor T-cells, which allows for tumor immune escape. The ability of the PD-1/PD-L1 axis to suppress antitumor immunity enables its application as a potential target for small-molecule-based immunotherapies. Targeting the PD-L1-mediated tumor immune evasion represents a promising approach for immune checkpoint blockade therapies. However, the existing monoclonal antibody-based therapies present poor overall response rates, warranting the development of small molecule drugs with the ability to regulate PD-L1 stability and enhance antitumor immunity. In this context, the present review summarizes the mechanisms of upstream PD-L1 regulation by kinases, cell cycle modulators, ubiquitin ligases, and glycosylation modulators, as well as the efficacy of small molecules targeting PD-L1 stability in regulating PD-L1-mediated immune evasion.
\end{abstract}

\section{Keywords}

Immunotherapy; PD-L1; small molecules; protein stability; post-translational modification

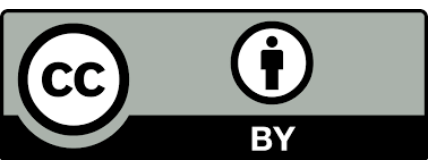

(C) 2021 by the author. This is an open access article distributed under the conditions of the Creative Commons by Attribution License, which permits unrestricted use, distribution, and reproduction in any medium or format, provided the original work is correctly cited. 


\section{Introduction}

Immune system evasion of the tumor microenvironment (TME) is characteristic of cancer and a critical biological property contributing to the etiology and progression of tumors [1]. The inhibitory immune checkpoint protein known as the programmed cell death protein 1 (PD-1) along with its ligand (PD-L1) has been implicated in the immunosuppressive behavior of the TME. PD-L1 is overexpressed in tumor cells, thereby creating an immunosuppressive state that allows these cells to escape T-cell mediated cytotoxicity [2]. One mechanism through which the tumor cells promote an immunosuppressive TME is the modulation of the tumor-antigen-specific T-cell activation [2]. In order to induce an antitumor immune response, the cytolytic $T$ lymphocytes must be activated into their effector state [2]. T-cell activation and their effector activity are strictly regulated by certain co-stimulatory and co-inhibitory receptors and ligands, among which PD-L1 forms a critical inhibitory checkpoint for cancer immunotherapies $[3,4]$. It is reported that PD-1 expression may also be induced in activated CD4+ and CD8+ T-lymphocytes and that the immunoinhibitory PD-1/PD-L1 axis supports self-tolerance and protects against autoimmunity [4, 5]. The PD-1/PD-L1 axis inhibits the survival and effector functions of T-lymphocytes, including proliferation, cytokine production, and cytolytic activity [4, 5]. However, chronic activation of the tumor-infiltrating lymphocytes expressing PD-1 may lead to an exhausted T-cell phenotype manifesting as the failure of T-cells to proliferate and exhibit their effector functions, such as cytokine production upon antigen exposure [6]. Therefore, therapeutic strategies targeting the PD-1/PD-L1 axis could be utilized to enhance the suppressed host immune response.

Recently, several monoclonal antibodies targeting the interaction between PD-1 and PD-L1 have been approved for clinical use with beneficial treatments in various cancer types, including melanomas, non-small-cell lung cancer (NSCLC), renal cell carcinoma (RCC), and bladder cancer [3, 7 , 8]. Even though the immune checkpoint antibodies have been applied successfully, their modest overall response rates and the development of therapeutic resistance following the initial effective response present scope for further improvement $[3,9,10]$. Moreover, the approved immunotherapies only include the monoclonal antibodies that target the PD-1-PD-L1 interactions, indicating a requirement for alternative therapeutic strategies targeting the PD-1/PD-L1 axis to improve the success rates of immune checkpoint blockade therapies (ICBT) [3, 11].

One of the promising therapeutic strategies targeting the PD-1/PD-L1 axis is the use of small molecules that regulate the PD-L1 stability to overcome the limitations of the antibody therapies. The benefits of using small molecules rather than monoclonal antibodies include reduced production costs and higher tumor penetration and drug stability owing to the lower molecular weight of these molecules $[12,13]$. Furthermore, higher amenability for oral administration and improved control over the half-life of these small molecules would result in a lower risk of immunogenic adverse effects that might otherwise occur with antibody therapies [14]. Further research and trials focused on small molecule-based drugs, and an improved understanding of the mechanisms influencing the stability of PD-L1 could assist in improving the efficacy of immune checkpoint blockade therapies. The present review was aimed to consolidate the current advancements in cancer immunotherapies based on small molecules targeting PD-L1 stability. 


\section{Regulation of PD-L1 Stability through Post-Translational Modifications}

Post-translational modifications (PTMs) are critical to the mechanisms involved in the regulation and control of protein structure, stability, and functions [15]. In particular, the mechanisms pertinent to PD-L1 stability include the influence of PTMs on the protein--protein interactions involved in degradation, cellular interactions, and communication with the cellular environment [15]. Important PTMs reported to be influencing the stability of PD-L1, and its subsequent immunosuppressive abilities include phosphorylation, glycosylation, and ubiquitination [16]. Incorporating changes in the oncogenic signaling pathways or the TME may facilitate antitumor immunity by affecting the cellular machinery regulating the PD-L1 PTMs [16]. Therefore, an improved understanding of the mechanisms regulating the PTMs of PD-L1 would enable the development of novel therapeutic targets for restoring antitumor immunity.

\subsection{Regulation of PD-L1 Stability through N-Glycosylation}

Enzymatic glycosylation of the extracellular domain is a post-translational modification that is critical to several membrane proteins and might serve as a therapeutic target for the small molecule modulators of PD-L1 stability [17]. The process of $N$-linked glycosylation of the extracellular domain commences within the endoplasmic reticulum (ER) with the enzymatic transfer of an oligosaccharide to an asparagine residue via the oligosaccharyltransferase complex [18]. Further processing and modifications of the core glycan continue within the Golgi apparatus and ensure protein integrity and the translocation of the mature PD-L1 protein to the cell surface [18]. Glycosylation plays an essential role in PD-L1 stability by ensuring proper protein folding, as the incorrectly folded proteins are targeted and degraded inside the ER [19]. Therefore, PD-L1 Nglycosylation represents an attractive target for small molecule-based drugs to alter the stability and the immunosuppressive function of PD-L1.

The primary isoform of PD-L1 is heavily glycosylated and weighs approximately $45 \mathrm{kDa}[20,21]$. PD-L1 undergoes $N$-linked glycosylation, and not the $O$-linked glycosylation, at four asparagine residues - N35, N192, N200, and N219 [20]. N-glycosylation is critical to the stability of PD-L1 as the non-glycosylated forms of PD-L1 are degraded relatively rapidly by the ubiquitin-proteasome system $[20,21]$. It is reported that the glycosylation of PD-L1, particularly at N192, N200, and $\mathrm{N} 219$, increases the protein stability, and the synchronous mutation of all three sites reduces the half-life of the protein to that of the non-glycosylated PD-L1 [20].

Tumor-associated alterations in the cellular machinery regulating PD-L1 glycosylation were demonstrated to modulate PD-L1 stability, expression, translocation, and immune evasion activity in pre-clinical models [16, 21-25]. FKBP51S, a co-chaperone protein of PD-L1, was demonstrated to enhance PD-L1 stability by binding to non-glycosylated PD-L1 within the ER and thereby catalyze the proper protein folding, a process determined to be necessary for glycosylation [22]. Sigma1, another chaperone protein of PD-L1 that is frequently upregulated in cancer cells, associates primarily with the glycosylated PD-L1 to promote proper protein folding, and the inhibition of Sigma1 decreases the levels of PD-L1 protein as well as its surface expression on the plasma membrane through enhanced protein degradation via selective autophagy [21]. Therefore, Sigma1-binding was demonstrated to prevent the degradation of PD-L1 by enhancing its stability and translocation to the plasma membrane in triple-negative breast and prostate cancer cells [21]. 
PD-L1 stability is also influenced by oncogenic alterations in the activity of $\mathrm{N}$ glycosyltransferase STT3A and $B$, the catalytic subunits of the oligosaccharyltransferase complex necessary for $N$-glycosylation [26]. An upregulation of STT3A/B in the cancer stem cells (CSCS) that had undergone epithelial-mesenchymal transition increased the PD-L1 protein levels, allowing for enhanced immune evasion [16]. The STT3 isoforms were observed to enhance PD-L1 stability evidenced by the significantly shorter half-life of the non-glycosylated PD-L1 in the STT3 isoform knockdown cells [16]. An increase in PD-L1 stability was also observed in response to upregulated STT3A/B with the increase in the $\beta$-Catenin expression in colon cancer cells, compared to that in the adjacent, non-cancerous tissues [27]. A recent study reported a positive association between the high expression of the epidermal growth factor receptor (EGFR) in triple-negative breast cancer cells and the expression of glycosyltransferase $\beta-1,3-\mathrm{N}$-acetylglucosaminyl transferase (B3GNT3) [23]. B3GNT3 was predicted to catalyze the addition of poly-LacNAc at the glycosylation sites N192 and N200 of PD-L1 [23]. Furthermore, the dietary polyphenol resveratrol (RSV) is thought to promote the abnormal, high-mannose glycosylation of PD-L1 by inhibiting the $N$ glycosylation-processing enzymes $\alpha$-glucosidase I and $\alpha$-mannosidase I [24]. The aberrant glycosylation of PD-L1 enhanced its endoplasmic reticulum-associated protein degradation (ERAD) $[24,25]$. Since the pharmacological inhibition of glycosylated PD-L1 enhances the internalization and degradation of PD-L1 [23], impeding PD-L1 glycosylation might serve as an effective strategy to overcome the PD-L1-mediated immunosuppression.

\subsection{Regulation of PD-L1 Stability through Phosphorylation}

Another promising approach for using novel small molecule inhibitors of PD-L1 is to target the upstream kinase and phosphatase regulators, which exert a dramatic influence on PD-L1 stability. Phosphorylation influences the PD-L1 stability primarily through the promotion of or interference with proper $N$-glycosylation, which is critical for PD-L1 stability as discussed earlier in Section 2.1. Glycogen synthase kinase $3 \beta$ (GSK3 $\beta$ ), a serine/threonine protein kinase, directly phosphorylates the non-glycosylated PD-L1 at the threonine residue 180 (T180) and serine residue 184 (S184) [20] and has been implicated as a tumor suppressor due to its involvement in the Wnt/ $\beta$-Catenin signaling pathway [28]. Li and colleagues (2016) further demonstrated that GSK3 $\beta$ exhibited antitumor activity through PD-L1 destabilization in human breast cancer cells. The sites of GSK3 $\beta$ mediated phosphorylation of PD-L1 were identified to be adjacent to the N192, N200, and N219 PD-L1 glycosylation sites [20]. Therefore, it was inferred that the phosphorylation at T180 and S184 could interfere with $N$-glycosylation due to steric hindrance. PD-L1 phosphorylation by GSK3 $\beta$ also recruits the E3 ligase named $\beta$-TrCP, which catalyzes PD-L1 ubiquitination and targets PD-L1 for degradation by 265 proteasome [20]. Therefore, phosphorylation by GSK3 $\beta$ destabilizes PD-L1 by promoting its cytoplasmic degradation, thereby representing a novel target for the small molecules modulating PD-L1 stability [20]. A second protein kinase that regulated the stability of PD-L1 via direct phosphorylation is the AMP-activated protein kinase (AMPK), which phosphorylates the extracellular domain of PD-L1 at S195 within the ER [25]. Phosphorylation of PD-L1 at S195 by AMPK results in the abnormal glycosylation of PD-L1, which could promote PD-L1 ERAD, as described earlier in Section 2.1 [25].

Recently, Chan and colleagues (2019) identified an interleukin-6 (IL-6)-activated Janus kinase 1 (JAK1), which is a non-receptor tyrosine kinase predicted to localize in the ER, to be serving as a 
mediator of PD-L1 stability. IL-6 was demonstrated to induce PD-L1 glycosylation by promoting the JAK1-mediated tyrosine phosphorylation of non-glycosylated PD-L1 at the tyrosine phosphorylation site, Y112, which enhanced PD-L1 association with, and glycosylation by, the ERassociated $\mathrm{N}$-glycosyltransferase STT3A [29]. Therefore, the proposed immunosuppressive mechanism underlying the increased IL-6 levels is the enhancement of JAK1-mediated phosphorylation and the subsequent STT3A-mediated glycosylation of PD-L1, which upregulates the expression of PD-L1 by enhancing its stabilization. In summary, the identification of the three kinases GSK3 $\beta$, AMPK, and JAK1 responsible for the phosphorylation of PD-L1 and the mechanisms regulating these kinases provide novel targets for PD-L1 immune checkpoint blockade therapies.

\subsection{Regulation of PD-L1 Stability by Ubiquitination}

Ubiquitination plays a critical role in the degradation of PD-L1, which emphasizes its importance in the stability of PD-L1 and its ability to mediate immune evasion. Ubiquitination is a complex post-translational modification that plays a significant role in the regulation of signaling proteins [30]. Ubiquitination involves the addition of ubiquitin via a covalent bond to the target proteins for the degradation of the latter by the $26 \mathrm{~S}$ proteasome, and the process is governed successively by ubiquitin-activating enzymes (E1s), ubiquitin-conjugating enzymes (E2s), and ubiquitin ligases (E3s) [30], which together form the ubiquitin--proteasome system (UPS), a critical regulatory system for cellular proteins. Dysregulation of the UPS, particularly the alterations or mutations influencing the E3 activity, could be contributing to the pathogenesis of cancers through the deregulation of the immune checkpoint control [30].

Multiple E3 ubiquitin ligases have been identified as the modulators of PD-L1 ubiquitination and degradation. PD-L1 ubiquitination by cullin-3 targets PD-L1 for degradation by the UPS [31]. However, the recruitment of cullin-3 and the subsequent ubiquitination of PD-L1 requires the binding of the speckle-type POZ protein (SPOP) to PD-L1 [31]. The loss-of-function mutations of SPOP or mutations in the PD-L1 SPOP binding site, therefore, improve PD-L1 stability [31]. As stated earlier, GSK3 $\beta$-mediated phosphorylation of PD-L1 causes the latter to associate with the E3 ligase $\beta$-TrCP, resulting in the ubiquitination of the phosphorylated-PD-L1 and its subsequent degradation by the $26 \mathrm{~S}$ proteasome [20]. A study reported that AMPK-mediated phosphorylation of PD-L1 at S195, which promotes its abnormal glycosylation, was followed by increased binding of PD-L1 to the ubiquitin ligase HRD1 [25]. The association of phosphorylated PD-L1 with HRD1 resulted in PD-L1 polyubiquitination, leading to ER-associated protein degradation (ERAD) [25]. Lastly, while the mechanisms underlying the STUB1-mediated ubiquitination of PD-L1 are relatively less known, STUB1 knockout in vivo decreased the PD-L1 polyubiquitination in the cells deficient in the PD-L1 stabilizer CMTM6 [32].

Upregulation of the PD-L1 stabilizing-molecules such as CMTM6 in tumor cells enhances the immunosuppressive abilities of PD-L1. While CMTM6 has not been reported to be involved in PDL1 trafficking, the flow cytometric and immunoprecipitation studies have demonstrated that CMTM6 protects PD-L1 from accelerated internalization by stabilizing its expression at the plasma membrane $[32,33]$. CMTM6 prevents the polyubiquitination of PD-L1 by the STUB1 E3 ligase, as evidenced by the increase in the PD-L1 protein levels upon STUB1 knockout in the CMTM6 knockdown melanoma cells [32]. Furthermore, CMTM6 promotes the endocytic recycling of PD-L1 
back to the cell surface after internalization, thereby preventing its degradation within the lysosome [33]. The secretion of the proinflammatory modulators TNF- $\alpha$ and CCL5 from the macrophages mediates PD-L1 stabilization through the upregulation of the deubiquitinase COP9 signalosome 5 (CSN5) [34, 35]. CSN5, in turn, reverses the PD-L1 ubiquitination to prevent protein degradation [34, 35]. The findings of Zhang and colleagues (2020) corroborate these results, as the authors demonstrated that the overexpression of the long non-coding RNA GATA3-AS1 in breast cancer cells enhances tumor immune evasion through the upregulation of CNS5, which then enhances the deubiquitination of PD-L1 [36].

Similarly, ubiquitin-specific peptidase 9 X-linked (USP9X) was reported to act as a deubiquitinase on PD-L1 to enhance the stability of the latter in oral squamous cell carcinoma [37]. USP9X reduced the PD-L1 ubiquitination and degradation, while the pharmacological inhibition of USP9X significantly reduced the half-life of PD-L1 through enhanced ubiquitination [37]. The identification of several pathways influencing PD-L1 ubiquitination emphasizes its important role as a post-translational modification regulating PD-L1 stability, thereby presenting new avenues for developing therapeutic small molecules targeting PD-L1.

\section{Regulation of PD-L1 Stability via Cell Cycle Modulators}

Cell cycle modulators play a significant role in PD-L1-mediated immune evasion, and their dysregulation reportedly leads to tumor development, due to which they are considered attractive targets for small molecule-based therapies [38]. Different stages of the cell cycle may lead to the modulation of PD-L1 stability and protein abundance [31]. Cyclin-dependent kinase 4 (CDK4), a regulator of the proteins involved in cell cycle progression, has been reported to decrease PD-L1 stability in vivo [31]. Mechanistically, CDK4 phosphorylates SPOP at serine 6, thereby stabilizing SPOP and promoting its binding to PD-L1, which enhances the ubiquitination of PD-L1 by the E3 ligase cullin-3, and ultimately, this polyubiquitination by cullin-3 targets PD-L1 for degradation [31] Cell-cycle progression alters the abundance of the SPOP protein and consequently decreases PDL1 stability, highlighting the role of cell-cycle modulators such as CDK4 as targets for small molecules for the regulation of PD-L1 stability.

\section{Regulation of PD-L1 Stability by Small Molecules}

As the understanding of the mechanisms underlying the regulation of PD-L1 stability is increasing, novel small molecule targets for immune blockage therapies are being identified. The majority of the small molecules identified to modulate PD-L1 stability evaluated in pre-clinical cell and animal models (summarized in Figure 1) have demonstrated encouraging results, promising their future application in clinical settings. Both synthetic and natural small molecule drugs have demonstrated therapeutic benefits and have been approved for the treatment of other human malignancies. Prior FDA approvals for these drugs and their success in regulating PD-L1 stability and improving the efficacy of immunotherapies in pre-clinical trials may ease the transition of these drugs into clinical trials. 


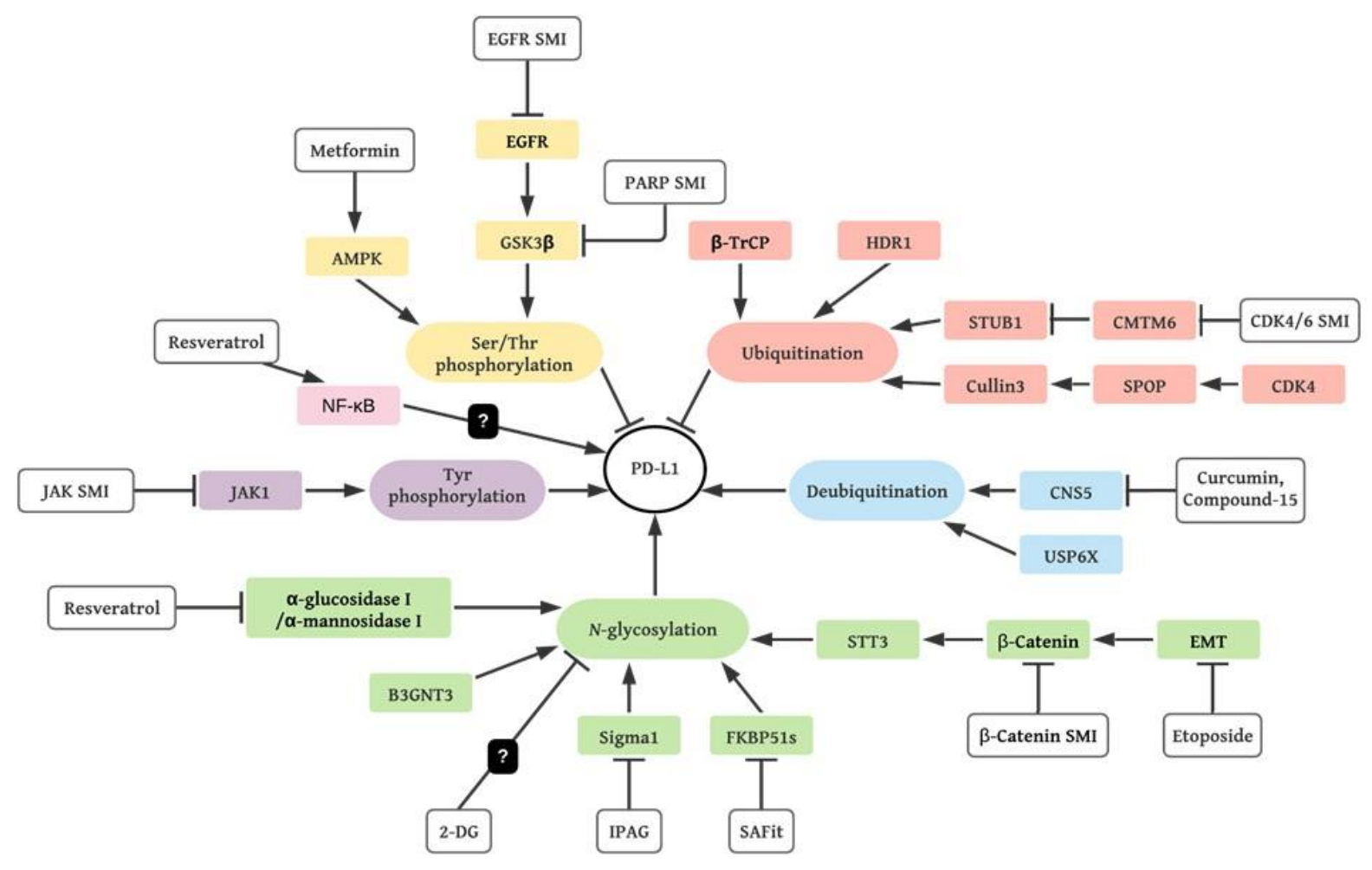

Figure 1 An overview of the cellular regulators affecting PD-L1 stability and the effects of small molecule drugs targeting these regulators.

\subsection{Small Molecules Increasing PD-L1 Stability}

Small molecule drugs with the ability to increase PD-L1 stability have the potential to further sensitize tumor cells to the currently available immune checkpoint blockade therapies. The current immunotherapies present low response rates and potential immunotoxicity, and the therapeutic enhancement of PD-L1 expression in the TME might increase the potency of these therapies by lowering the required therapeutic dose and the corresponding adverse effects on health.

CDK4 is recognized as a negative regulator of PD-L1 stability [31] and, therefore, the smallmolecule inhibitors (SMIs) of the CDK4/6 kinase complex are being successfully used in pre-clinical trials to enhance the response to immune checkpoint blockade [39, 40]. Two selective SMIs of CDK4/6, palbociclib and ribociclib, when used for the treatment of cancer cell lines, increased both PD-L1 stability and protein levels [31], and this increase in the PD-L1 stability enhanced the tumor responses to immune blockade therapies [31]. Palbociclib and trilaciclib, two FDA-approved selective SMIs of CKD4/6, could augment the antitumor T-cell response and almost completely inhibited the tumor growth with the use of PD-1 blockade antibodies in murine cancer models and human tumor samples [41]. Furthermore, the combined use of the CDK4/6 SMI abemaciclib and anti-PD-L1 in murine cancer models resulted in a greater decrease in tumor growth compared to that when using either of the therapies independently, and also inhibited the continuation of growth after the initial halt [40]. Therefore, the synergistic strategy of using CDK4/6 inhibitors in combination with the current ICBTs may enhance antitumor immunity by promoting PD-L1 stabilization.

PARP inhibitors (PARPi) have recently been demonstrated to have potential application in cotreatment with ICBTs to increase the treatment efficacy and to prevent tumor resistance to 
therapies [41]. PARPi are already approved for the treatment of ovarian cancer, and their influence on PD-L1 stability demonstrates their potential to deal with tumor immune resistance. Triple-negative breast cancer cells and in vivo models treated with the PARP SMIs olaparib and talazoparib exhibit significantly increased PD-L1 expression at the plasma membrane [41]. PARPi was demonstrated to inactivate GSK3 $\alpha / \beta$, which increased PD-L1 stability as GSK3 $\beta$-mediated phosphorylation promotes PD-L1 degradation $[41,20]$. Olaparib was reported to sensitize murine tumors to PD-L1 blockade, resulting in decreased tumor growth and the restoration of CD8+ T-cell levels [41]. The combination therapies demonstrated higher efficacy, with no observed toxicity, compared to either of the therapies alone [41].

While studies on the influence of resveratrol (RSV) on PD-L1 stability have reported inconsistent findings, Lucas and colleagues (2018) reported that the treatment of certain breast and colorectal cancer cells with the stilbenoids RSV and piceatannol increased the PD-L1 protein abundance. RSV and piceatannol were predicted to act by upregulating NF-kB, which is intricately involved in the transcription of PD-L1 [42]. Furthermore, the tumors expressing low levels of PD-L1 mRNA were observed to exhibit higher sensitivity to RSV and/or piceatannol, indicating the potential of these drugs for the therapeutic sensitization of resistant tumors to ICBT [42]. Since an intensive treatment has the potential to enhance PD-L1-mediated tumor immune evasion, determining the clinically relevant doses and the duration of treatment is critical [43]. However, higher overall response rates to anti-PD-L1 blockade are observed in PD-L1-positive tumors [43]. Therefore, these small molecules modulating PD-L1 stability and expression could be employed to enhance the therapeutic efficacy of the currently available treatments.

\subsection{Small Molecules Decreasing PD-L1 Stability}

Small molecules decreasing PD-L1 stability have the potential to enhance the efficacy, potency, and safety of immune checkpoint blockade therapies. Similar to small molecules that enhance the PD-L1 stability, those decreasing the PD-L1 stability are promising as additive therapeutic agents with the potential for application in immune checkpoint blockade monotherapies that have demonstrated little success so far.

\subsubsection{Small Molecules Decreasing PD-L1 Stability by Regulating PD-L1 Phosphorylation}

Since recent pre-clinical studies have demonstrated the importance of kinases and phosphorylation in influencing PD-L1 stability, small molecules targeting the pathways regulating PD-L1 phosphorylation are being evaluated as therapeutic agents. A few such small molecules are described below.

Metformin. Metformin was initially known as a promotor of antitumor activity acting through a relatively unknown mechanism, until Cha and colleagues (2018) identified AMPK as the effector of metformin's antitumor immunity abilities. It has been demonstrated that metformin exposure activates AMPK, which then phosphorylates PD-L1 at S195 to induce the ERAD of the latter [25]. Metformin treatment could reduce PD-L1 stability and expression in vivo in murine cancer models and human breast cancer tumor tissues [25]. When metformin treatment was combined with blockage of CTLA4, which is another clinically significant immune checkpoint protein, tumor growth and survival rate were significantly enhanced without any observable toxicity [25]. These 
findings provide a rationale for a mechanism-driven approach to block the immune inhibitor signaling of PD-L1, either independently or in combination with the other immune checkpoint blockers.

EGF Inhibitors. Since EGF stimulation increases PD-L1 stability by inhibiting the phosphorylation of PD-L1 by GSK3 $\beta$, the use of an EGF receptor (EGFR) inhibitor gefitinib predictably decreased the PD-L1 stability via the same mechanism [20]. In vivo treatment of basal-like breast cancer (BLBC) cells with gefitinib decreased the PD-L1 expression in syngeneic mouse models [20]. Increased phosphorylation by GSK3 $\beta$ inhibited the proper $N$-glycosylation of PD-L1, thereby decreasing PDL1 stability. Gefitinib treatment also sensitized the tumor cells to anti-PD-1 antibody treatment [20]. Kim et al. (2020) assessed the effects of the EGFR inhibitor gefitinib along with a saccharide analog 2-deoxy-d-glucose (2-DG) and reported that gefitinib and 2-DG were synergistic inhibitors of PD-L1 glycosylation [44]. Although metabolic pathways are implicated in the regulation of PD-L1 glycosylation, the authors predicted that 2-DG acted in a glycolysis-independent manner [44]. The use of gefitinib and 2-DG in combination with an agonist antibody of the 4-1BB co-stimulatory receptor present on T-cells synergistically ameliorated the antitumor activity of cytotoxic T-cells in murine models [44]. Since 2-DG and the- agonist antibodies of 4-1BB, when used individually, have not proven to be sufficiently efficacious to validate their toxic effects, using them in lower doses in combination with gefitinib might serve as an alternate immune checkpoint therapy $[45,46]$.

JAK1 Inhibitor. Inhibition of JAK1 with ruxolitinib, a selective small molecule inhibitor of JAK1/2 kinase, was observed to decrease PD-L1 stability in the cancer cells stimulated by IL-6 [29]. Ruxolitinib inhibited the IL-6-induced JAK1-mediated phosphorylation of PD-L1 at Y112, thereby increasing the PD-L1 ubiquitination and the turnover rate in hepatocellular carcinoma cells [29]. Ruxolitinib already has the FDA approval for use in certain myeloproliferative diseases, which might ease its integration into future pre-clinical and clinical trials targeting the immune evasion mediated by PD-L1 [29]. Small molecule inhibition of the phosphorylation of PD-L1 at Y112 by using ruxolitinib also interfered with the recruitment of STT3A, which is required to stabilize PD-L1 via proper $N$-glycosylation [29]. Other small molecules have also been evaluated for targeting this downstream regulator. $\beta$-Catenin is reported to positively regulate $N$-glycosyltransferase STT3A/B, thereby allowing for immune evasion in colon cancer stem cells (CSCs) [27]. Exposure to the $\beta$ Catenin SMI KYA1797K decreased its ability to transactivate STT3A/B, thereby inhibiting PD-L1 Nglycosylation and stabilization [27]. Furthermore, etoposide, a topoisomerase II poison reported to be moderately selective of CSCS, inhibits the EMT/ $\beta$-catenin/STT3/PD-L1 axis by decreasing STT3 expression, thereby reducing the protein levels of glycosylated PD-L1 [16, 47]. In murine cancer models, while the use of etoposide alone did not significantly restore the CD8+ T-cell-mediated cytotoxicity, it did function as an additive with the anti-Tim-3 blockade therapy to induce T-cell cytotoxicity and reduce tumor burden [16]. These findings highlight the potential of the upstream inhibition of PD-L1 $\mathrm{N}$-linked glycosylation by STT3 as an immunotherapeutic agent to be used in combination therapies.

\subsubsection{Small Molecules Decreasing PD-L1 Stability by Regulating Glycosylation}

Several small molecules reduce PD-L1 stability by regulating the glycosylation of PD-L1 in a phosphorylation-independent manner. 
Sigma1 Inhibitor. Small molecule inhibition of Sigma1 using IPAG decreased the PD-L1 protein levels in TNBC cells and androgen-independent prostate cancer cells [21]. Sigma1 is a chaperone protein of PD-L1 and, therefore, promotes the stability and trafficking of glycosylated PD-L1. Sigma1 inhibition using IPAG reportedly induced autophagic degradation of PD-L1 via interference with post-translational modifications, as evidenced in the treatment of cells with IPAG, which decreased protein levels [21].

FKBP51S Inhibitors. Selective small molecule inhibitors of FKBP51S, namely, SAFit 1 and SAFit 2, were also demonstrated to modulate the post-translational modifications by impeding PD-L1 glycosylation, which led to decreased PD-L1 plasma membrane protein levels in glioblastoma cell lines [22]. In another study, SAFit2 treatment significantly decreased PD-L1 expression and consequently the tumor growth, in addition to negating the upregulation of PD-L1 expression upon ionizing radiation, which is frequently used as a glioma treatment [48]. Consequently, the SMIs of FKBP51S are considered attractive targets to relieve the PD-L1-induced immune evasion, particularly in the tumors with upregulated PD-L1 protein levels upon radiation treatment.

Curcumin. Curcumin, a CSN5 inhibitor, inhibits the TNF- $\alpha$-mediated stabilization of PD-L1 in various cancer cell types, including melanoma, breast, colon, and lung cancers [35]. Inhibition of the PD-L1 deubiquitinase CNS5 using curcumin diminished tumor growth and served as an additive that could enhance the efficacy of CTLA4 immune blockade [35]. Another study assessing a SMI of CNS5, compound-15, reported decreased PD-L1 stabilization and reduced tumor growth [34].

BMS-1166 and Other Inhibitors from Bristol Myers Squibb. Bristol Myers Squibb (BMS) has developed and patented [49] a series of small molecule inhibitors that target PD-L1 and bind to the region of PD-L1 that interfaces with PD-1 [50,51], although the inhibitory mechanism is not thought to involve a direct inhibition of PD-L1/PD-1 interaction and rather involves the induction of PD-L1 dimerization by the binding of these small molecules $[50,51]$. A recent study focusing on BMS-1166, which has a particularly low IC50 value of $1.4 \mathrm{nM}$, further characterized this mechanism of inhibition [52]. BMS-1166 treatment specifically affected the PD-L1 glycosylation by preventing the export of PD-L1 from the ER to the Golgi apparatus. In addition, the underglycosylated form of PD-L1 was observed to undergo rapid degradation in the ER [52]. Taken together, these findings suggested a mechanism in which the treatment with BMS-1166 induces dimerization and sequestration of PD-L1 within the ER, thereby preventing further glycosylation and leading to ERAD. Furthermore, BMS-1166 was demonstrated to prevent T-cell inactivation by PD-L1/PD-1 interaction in a co-culture of lung cancer cells and $T$ cells. It is noteworthy that the specificity of these compounds for PD-L1 minimizes the off-target effects and, therefore, provides a promising strategy for small molecule immunotherapies.

RSV. RSV has been reported to increase PD-L1 stability in previous studies, although with contradictory results. Verdura and colleagues (2020) employed a computational approach to predict that RSV interferes with PD-L1 stability and plasma membrane trafficking via two mechanisms [24]. In breast cancer cells, RSV may inhibit PD-L1 N-glycosylation processing enzymes $\alpha$-glucosidase I and $\alpha$-mannosidase I, thereby resulting in the abnormal glycosylation of PD-L1 [24]. On the other hand, RSV may interact with the PD-L1/PD-1 binding site, thereby promoting the dimerization of PD-L1 similar to the series of compounds developed by Bristol Myers Squibb [24]. 
Another study reported that RSV treatment promoted immune cell activation while also leading to ovarian carcinoma cell death independent of its effects on immune cells [53]. Overall, RSV appears to function similar to the BMS compounds, i.e., by inducing PD-L1 dimerization, although it may also exert other effects that directly or indirectly influence the PD-L1 stability. Further characterization of the mechanisms underlying the RSV action, including the investigation of offtarget effects, is required prior to considering this compound as a viable small molecule inhibitor for cancer immunotherapy.

\section{Conclusion}

Accumulating evidence suggests that the complex mechanisms regulating PD-L1 stability are altered in cancer to allow for tumor immune evasion. Therefore, the cellular modulators of PD-L1 stability may provide novel small molecule targets for disrupting the immunosuppressive capacity of PD-L1 in the TME. The currently available clinical PD-L1 immune blockade checkpoint therapies frequently present low overall response rates, primary or acquired resistance, and/or immunotoxicity. Therefore, to overcome these issues and improve patient prognosis, pre-clinical studies have begun preferring the use of small molecule inhibitors in mono- or combination therapies with antibody immune blockade. Furthermore, several of the small molecules identified to modulate PD-L1 stability have already received approval for use in clinical settings at therapeutic doses and may prove to be successful in increasing the potency of the existing immune checkpoint therapies while preventing the adverse effects. Therefore, small molecules targeting PD-L1 stability represent a promising new generation of immune checkpoint blockade therapies.

\section{Author Contributions}

M.L. and A.P wrote the manuscript and X.Y. revised the manuscript.

\section{Funding}

Canadian Institute of Health Research (CIHR\#119325, 148629), Canadian Breast Cancer Foundation/Canadian Cancer Society (CBCF/CCS) to X.Y.

\section{Competing Interests}

The authors have declared that no competing interests exist.

\section{References}

1. Hanahan D, Weinberg RA. Hallmarks of cancer: The next generation. Cell. 2011; 144: 646-674.

2. Jiang $X$, Wang J, Deng $X$, Xiong $F, G e J$, Xiang $B$, et al. Role of the tumor microenvironment in PD-L1/PD-1-mediated tumor immune escape. Mol Cancer. 2019; 18: 10.

3. Wang Y, Wang H, Yao H, Li C, Fang JY, Xu J. Regulation of PD-L1: Emerging routes for targeting tumor immune evasion. Front Pharmacol. 2018; 9: 536.

4. Li K, Tian H. Development of small-molecule immune checkpoint inhibitors of PD-1/PD-L1 as a new therapeutic strategy for tumour immunotherapy. J Drug Target. 2019; 27: 244-256. 
5. Zitvogel L, Kroemer G. Targeting PD-1/PD-L1 interactions for cancer immunotherapy. Oncoimmunology. 2012; 1: 1223-1225.

6. Pauken KE, Wherry EJ. Overcoming T cell exhaustion in infection and cancer. Trends Immunol. 2015; 36: 265-276.

7. Akinleye A, Rasool Z. Immune checkpoint inhibitors of PD-L1 as cancer therapeutics. J Hematol Oncol. 2019; 12: 92.

8. Zhou $\mathrm{Y}$, Lin Z, Zhang $\mathrm{X}$, Chen $\mathrm{C}$, Zhao H, Hong S, et al. First-line treatment for patients with advanced non-small cell lung carcinoma and high PD-L1 expression: Pembrolizumab or pembrolizumab plus chemotherapy. J Immunother Cancer. 2019; 7: 120.

9. Sharma P, Hu-Lieskovan S, Wargo JA, Ribas A. Primary, adaptive, and acquired resistance to cancer immunotherapy. Cell. 2017; 168: 707-723.

10. Brahmer JR, Tykodi SS, Chow LQ, Hwu WJ, Topalian SL, Hwu P, et al. Safety and activity of antiPD-L1 antibody in patients with advanced cancer. N Engl J Med. 2012; 366: 2455-2465.

11. Lee HT, Lee SH, Heo YS. Molecular interactions of antibody drugs targeting PD-1, PD-L1, and CTLA-4 in immuno-oncology. Molecules. 2019; 24: 1190.

12. Skalniak L, Zak KM, Guzik K, Magiera K, Musielak B, Pachota M, et al. Small-molecule inhibitors of PD-1/PD-L1 immune checkpoint alleviate the PD-L1-induced exhaustion of T-cells. Oncotarget. 2017; 8: 72167-72181.

13. Kerr WG, Chisholm JD. The next generation of immunotherapy for cancer: Small molecules could make big waves. J Immunol. 2019; 202: 11-19.

14. Zhan MM, Hu XQ, Liu XX, Ruan BF, Xu J, Liao C. From monoclonal antibodies to small molecules: The development of inhibitors targeting the PD-1/PD-L1 pathway. Drug Discov Today. 2016; 21: 1027-1036.

15. Wang YC, Peterson SE, Loring JF. Protein post-translational modifications and regulation of pluripotency in human stem cells. Cell Res. 2014; 24: 143-160.

16. Hsu JM, Li CW, Lai YJ, Hung MC. Posttranslational modifications of PD-L1 and their applications in cancer therapy. Cancer Res. 2018; 78: 6349-6353.

17. Chandler KB, Costello CE. Glycomics and glycoproteomics of membrane proteins and cell-surface receptors: Present trends and future opportunities. Electrophoresis. 2016; 37: 1407-1419.

18. Schwarz F, Aebi M. Mechanisms and principles of $\mathrm{N}$-linked protein glycosylation. Curr Opin Struct Biol. 2011; 21: 576-582.

19. Breitling J, Aebi M. N-linked protein glycosylation in the endoplasmic reticulum. Cold Spring Harb Perspect Biol. 2013; 5: a013359.

20. Li CW, Lim SO, Xia W, Lee HH, Chan LC, Kuo CW, et al. Glycosylation and stabilization of programmed death ligand-1 suppresses T-cell activity. Nat Commun. 2016; 7: 12632.

21. Maher CM, Thomas JD, Haas DA, Longen CG, Oyer HM, Tong JY, et al. Small-molecule sigma1 modulator induces autophagic degradation of PD-L1. Mol Cancer Res. 2018; 16: 243-255.

22. D’Arrigo P, Russo M, Rea A, Tufano M, Guadagno E, De Caro ML, et al. A regulatory role for the co-chaperone FKBP51s in PD-L1 expression in glioma. Oncotarget. 2017; 8: 68291.

23. Li CW, Lim SO, Chung EM, Kim YS, Park AH, Yao J, et al. Eradication of triple-negative breast cancer cells by targeting glycosylated PD-L1. Cancer Cell. 2018; 33: 187-201.e10.

24. Verdura S, Cuyàs E, Cortada E, Brunet J, Lopez-Bonet E, Martin-Castillo B, et al. Resveratrol targets PD-L1 glycosylation and dimerization to enhance antitumor T-cell immunity. Aging. 
2020; 12: 8-34.

25. Cha JH, Yang WH, Xia W, Wei Y, Chan LC, Lim SO, et al. Metformin promotes antitumor immunity via endoplasmic-reticulum-associated degradation of PD-L1. Mol Cell. 2018; 71: 606-620.

26. Lara P, Öjemalm K, Reithinger J, Holgado A, Maojun Y, Hammed A, et al. Refined topology model of the STT3/Stt3 protein subunit of the oligosaccharyltransferase complex. J Biol Chem. 2017; 292: 11349-11360.

27. Ruan Z, Liang M, Lai M, Shang L, Deng X, Su X. KYA1797K down-regulates PD-L1 in colon cancer stem cells to block immune evasion by suppressing the $\beta$-catenin/STT3 signaling pathway. Int Immunopharmacol. 2020; 78: 106003.

28. Wu D, Pan W. GSK3: A multifaceted kinase in Wht signaling. Trends Biochem Sci. 2010; 35: 161-168.

29. Chan LC, Li CW, Xia W, Hsu JM, Lee HH, Cha JH, et al. IL-6/JAK1 pathway drives PD-L1 Y112 phosphorylation to promote cancer immune evasion. J Clin Invest. 2019; 129: 3324-3338.

30. Morrow JK, Lin HK, Sun SC, Zhang S. Targeting ubiquitination for cancer therapies. Future Med Chem. 2015; 7: 2333-2350.

31. Zhang J, Bu X, Wang H, Zhu Y, Geng Y, Nihira NT, et al. Cyclin D-CDK4 kinase destabilizes PD-L1 via cullin 3-SPOP to control cancer immune surveillance. Nature. 2018; 553: 91-95.

32. Mezzadra R, Sun C, Jae LT, Gomez-Eerland R, De Vries E, Wu W, et al. Identification of CMTM6 and CMTM4 as PD-L1 protein regulators. Nature. 2017; 549: 106-110.

33. Burr ML, Sparbier CE, Chan YC, Williamson JC, Woods K, Beavis PA, et al. CMTM6 maintains the expression of PD-L1 and regulates anti-tumour immunity. Nature. 2017; 549: 101-105.

34. Liu C, Yao Z, Wang J, Zhang W, Yang Y, Zhang Y, et al. Macrophage-derived CCL5 facilitates immune escape of colorectal cancer cells via the p65/STAT3-CSN5-PD-L1 pathway. Cell Death Differ. 2020; 27: 1765-1781.

35. Lim SO, Li CW, Xia W, Cha JH, Chan LC, Wu Y, et al. Deubiquitination and stabilization of PD-L1 by CSN5. Cancer Cell. 2016; 30: 925-939.

36. Zhang $M$, Wang $N$, Song $P, F u ~ Y$, Ren $Y$, Li Z, et al. LncRNA GATA3-AS1 facilitates tumour progression and immune escape in triple-negative breast cancer through destabilization of GATA3 but stabilization of PD-L1. Cell Prolif. 2020; 53: e12855.

37. Jingjing W, Wenzheng G, Donghua W, Guangyu H, Aiping Z, Wenjuan W. Deubiquitination and stabilization of programmed cell death ligand 1 by ubiquitin-specific peptidase $9, \mathrm{X}$-linked in oral squamous cell carcinoma. Cancer Med. 2018; 7: 4004-4011.

38. Otto T, Sicinski P. Cell cycle proteins as promising targets in cancer therapy. Nat Rev Cancer. 2017; 17: 93-115.

39. Goel S, DeCristo MJ, Watt AC, BrinJones H, Sceneay J, Li BB, et al. CDK4/6 inhibition triggers anti-tumour immunity. Nature. 2017; 548: 471-475.

40. Deng J, Wang ES, Jenkins RW, Li S, Dries R, Yates K, et al. CDK4/6 inhibition augments antitumor immunity by enhancing T-cell activation. Cancer Discov. 2018; 8: 216-233.

41. Jiao S, Xia W, Yamaguchi H, Wei Y, Chen MK, Hsu JM, et al. PARP inhibitor upregulates PD-L1 expression and enhances cancer-associated immunosuppression. Clin Cancer Res. 2017; 23: 3711-3720.

42. Lucas J, Hsieh TC, Halicka HD, Darzynkiewicz Z, Wu JM. Upregulation of PD-L1 expression by resveratrol and piceatannol in breast and colorectal cancer cells occurs via HDAC3/p300- 
mediated NF-KB signaling. Int J Oncol. 2018; 53: 1469-1480.

43. Chen $Q$, Li T, Yue W. Drug response to PD-1/PD-L1 blockade: Based on biomarkers. OncoTargets Ther. 2018; 11: 4673-4683.

44. Kim B, Sun R, Oh W, Kim AM, Schwarz JR, Lim SO. Saccharide analog, 2-deoxy-d-glucose enhances 4-1BB-mediated antitumor immunity via PD-L1 deglycosylation. Mol Carcinog. 2020; 59: 691-700.

45. Chester C, Sanmamed MF, Wang J, Melero I. Immunotherapy targeting 4-1BB: Mechanistic rationale, clinical results, and future strategies. Blood. 2018; 131: 49-57.

46. Singh D, Banerji AK, Dwarakanath BS, Tripathi RP, Gupta JP, Mathew TL, et al. Optimizing cancer radiotherapy with 2-deoxy-D-glucose. Strahlenther Onkol. 2005; 181: 507-514.

47. Gupta PB, Onder TT, Jiang G, Tao K, Kuperwasser C, Weinberg RA, et al. Identification of selective inhibitors of cancer stem cells by high-throughput screening. Cell. 2009; 138: 645659.

48. D’Arrigo P, Digregorio $M$, Romano $S$, Tufano $M$, Rea A, Hausch F, et al. The splicing FK506binding protein-51 isoform plays a role in glioblastoma resistance through programmed cell death ligand-1 expression regulation. Cell Death Discov. 2019; 5: 137.

49. Chupak LS, Zheng X. Compounds useful as immunomodulators. New York, NY: Bristol-Myers Squibb Co; 2015; WO2015034820A1.

50. Zak KM, Grudnik P, Guzik K, Zieba BJ, Musielak B, Dömling A, et al. Structural basis for small molecule targeting of the programmed death ligand 1 (PD-L1). Oncotarget. 2016; 7: 3032330335.

51. Guzik K, Zak KM, Grudnik P, Magiera K, Musielak B, Törner R, et al. Small-molecule inhibitors of the programmed cell death-1/programmed death-ligand 1 (PD-1/PD-L1) interaction via transiently induced protein states and dimerization of PD-L1. J Med Chem. 2017; 60: 58575867.

52. Chen FF, Li Z, Ma D, Yu Q. Small-molecule PD-L1 inhibitor BMS1166 abrogates the function of PD-L1 by blocking its ER export. Oncoimmunology. 2020; 9: 1831153.

53. Zhang $Y$, Yang $S$, Yang $Y$, Liu T. Resveratrol induces immunogenic cell death of human and murine ovarian carcinoma cells. Infect Agents Cancer. 2019; 14: 27.

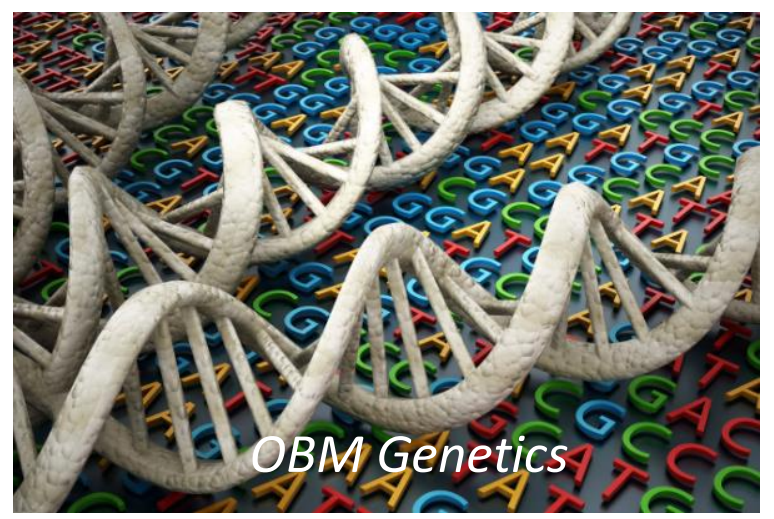

Enjoy OBM Genetics by:

1. Submitting a manuscript

2. Joining in volunteer reviewer bank

3. Joining Editorial Board

4. Guest editing a special issue

For more details, please visit: http://www.lidsen.com/journals/genetics 